\title{
TARGET VALUE DESIGN: DEVELOPMENT AND TESTING OF A VIRTUAL SIMULATION
}

\author{
Georgie Jacob $^{1}$, Nimish Sharma ${ }^{2}$, Zofia K. Rybkowski ${ }^{3}$, and Ganesh Devkar ${ }^{4}$
}

\begin{abstract}
Early in the development of a lean project, Target Value Design (TVD) practices define owner value, and it is toward actualization of defined owner value that all subsequent lean practices should flow. Participatory simulations have been used to help stakeholders comprehend TVD processes before they are implemented on an actual project, enhancing their effectiveness. This paper introduces results from testing of an online version of a TVD simulation that was being used to teach TVD at universities and to practioner stakeholders before embarking on a sometimes lengthy TVD journey. The online TVD simulation described in this paper arose out of the need to continue to teach TVD despite social distancing requirements that emerged during the global COVID-19 pandemic. This paper chronicles the details associated with the online simulation: the template design, choice of suitable online platform, strategy for playing the simulation, and facilitation of post-simulation discussions. The developed simulation was tested with post graduate students of Construction Engineering and Management Programme at CEPT University. The post simulation discussion and analysis of questionnaire responses received indicate that participants enjoyed this simulation and learned important principles related to TVD. This online simulation is an evolved version of the Marshmallow Tower TVD simulation. Hence, it indicates the growing trend towards evolution of lean simulations and serious game to adjust to changing conditions.
\end{abstract}

\section{KEYWORDS}

Target Value Delivery, collaboration, target cost, virtual online simulation, lean simulation.

\section{INTRODUCTION}

Experimentation with serious games and simulations to instil the concepts and principles of lean construction among students and practitioners has been explored since the early 1980s by prominent researchers such as Greg Howell and Glenn Ballard (Rybkowski et al. 2020). Rybkowski et al. (2020) compiled a list of lean simulations facilitated by twelve academicians at major universities to impart specific lean principles. Additionally, forums such as the Lean Construction Institute (LCI) and the International Group of Lean

1 Teaching Associate, Faculty of Technology, CEPT University, Ahmedabad - 380009, India +919961957876, georgie.jacob@ cept.ac.in, orcid.org/0000-0001-6548-9538

2 Post Graduate Student, Faculty of Technology, CEPT University, Ahmedabad - 380009, India +918368938579, nimish.sharma@cept.ac.in, orcid.org/0000-0002-8050-7893

3 Associate Proffessor, Department of Construction Science, Texas A\&M University, College Station, TX 77843, USA, zrybkowski@tamu.edu, orcid.org/0000-0002-0683-5004

4 Associate Professor, Faculty of Technlogy, CEPT University, Ahmedabad - 380009, India +919099010303, ganesh.devkar@cept.ac.in, orcid.org/0000-0002-5482-1221 
Construction (IGLC) have been extensively using and encouraging lean games and simulations to motivate adoption of lean practices across the construction supply chain. The Target Value Design (TVD) simulation inspired by the "marshmallow design challenge" (Skillman 2014) is one such lean construction simulation (Rybkowski et al. 2016), which imparts the principles of the Lean-Integrated Project Delivery.

The COVID-19 pandemic severely impacted the ability of educators to facilitate lean simulation and games that were previously played in-person in a physical space. Globally, the lean community adapted to this situation by transforming and transplanting these games and simulations to the virtual realm.

This paper describes the development and testing of an improvised and transformed version of the marshmallow tower TVD simulation which can be played virtually online.

\section{TVD SIMULATION}

Target Value Design (TVD) evolved from target costing or genka kikaku. TVD involves continuous, collaborative cross-disciplinary appraisal of design proposals to improve overall value through the lens of various constraints such as cost, design, risk, constructability, quality, and time (Lee et al. 2012).

Experimentation of TVD practices and their outcomes on construction projects have been documented (Ballard and Rybkowski 2009). Do et al. (2014) state that TVD practices can help stakeholders successfully achieve a target cost that is $15 \%$ to $20 \%$ below market cost while maximizing the overall value to the project and owner. Early collaboration of stakeholders, setting a target cost and rigorous estimating are key requirements of TVD, as described by Ballard (2008).

The TVD simulation or marshmallow tower TVD simulation, developed by Munankami and Rybkowski (Rybkowski et al., 2016), was based on the "marshmallow design challenge" game by Peter Skillman. It has become widely used by university educators and lean consultants to impart principles of TVD, such as market cost, target cost, allowable cost, estimated cost, collaboration, etc. among the participants. It requires the groups of participants to design and then build a freestanding 2-feet tall tower that is no more than 2 inches out-of-plumb, using materials provided by the facilitator. Participants are then asked to optimize the design of the tower to lower the total cost by $15 \%$ to $20 \%$ without losing the overall value criteria established by the building owner (Rybkowski et al. 2020). The popularity of the TVD simulation may be due, in part, to the fact that it can be played in as little as 50 minutes, with relatively inexpensive materials. In fact, the simulation continues to be transformed following analyses and "plus delta" feedback from participants. For example, Devkar et al. (2019) had developed and tested a TVD simulation which includes BIM for visualization and rapid cost feedback. The virtual TVD simulation in this paper is an improvised version of the marshmallow tower TVD simulation which can be played virtually. This virtual TVD simulation was developed and tested at CEPT University.

\section{SIMULATION DEVELOPMENT}

\section{INSTRUCTIONS TO PLAY}

The objective of this online TVD simulation was to provide to participants a first-hand experience of the fundamental goals of Target Value Design. The game is inspired from TVD marshmallow tower game developed by Munankami and Rybkowski (Munankami 2012; Rybkowski et al. 2016), which is a widely played simulation administered in person 
prior to the COVID-19 pandemic. As part of a studio course named "Construction Project Formulation and Appraisal" for graduate students of Construction Engineering and Management Programme at CEPT University, the TVD marshmallow tower game had been incorporated into the course curriculum. However, during the COVID-19 global pandemic, classrooms and studios were moved to an online environment. The instructor for this studio course faced the challenge of transforming the existing TVD simulation, typically played in the physical realm, into a virtual simulation. This pressing need resulted in the crafting and testing of the online TVD simulation described in this paper.

The online TVD simulation developed by this team involved the design, construction and costing of a tower having height and base width requirements of $26 \mathrm{~cm}$ and $12 \mathrm{~cm}$ respectively. Online game templates were prepared for the students to play the simulation. The task of each team was to construct a tower with the help of shapes provided in the game template. The shapes and their three size variants are shown in Figure 1. Typically, a team of 3-4 participants was required to play this simulation. Each team member was asked to select and assume either of following roles: Designer, Contractor, or Owner. Based on the number of participants playing this simulation, two participants were allowed to play the role of Owner. However, the roles of Designer and Contractor were played by a single participant only. The simulation comprised two rounds. Round 1 modelled the traditional mode of project delivery, simulating a design-bid-build model of siloed operations along the construction supply chain, while the Round 2 simulated the type of active simultaneous collaboration required during TVD. In Round 1, the Owner was asked to prepare a strategic brief and then hand it over to the Designer. While preparing the strategic brief, the Owner could select shapes and the general aesthetics of the tower, keeping in mind specifications pertaining to the height and base width of the tower. The Designer was then asked to prepare a design of tower (Design Proposal- D1) according to requirements stated in the strategic brief prepared by the Owner. The design needed to be approved by the Owner before being handed over to the Contractor. The Contractor was then expected to construct the tower in accordance with the Design Proposal - D1, using shapes provided in the game template. Verbal communication between the Owner, Designer and Contractor was restricted in the first round. Communication between each role-play was limited to Requests for Information (RFIs) and needed to be continually documented. Although, specific time limits were not enforced for Round 1, the teams were pressed to finish as soon as possible. Pressure took the form of online streaming quotes such as "Time is the Essence of the Contract" while playing the online simulation. After the completion of Round 1, the teams were asked to calculate the cost of their constructed towers (Cost $-\mathrm{C} 1)$. Further to this, each team had to declare its target cost (Target Cost - T1). In contrast to Round 1, Round 2 was designed as a collaborative environment where team members could collaborate and openly communicate with one other without any restrictions, beginning from design. In this round, the teams were expected to design (Design - D2) and construct their towers, keeping in mind the declared target cost, while aspiring to construct them at costs even lower than their declared target costs. At the completion of Round 2, teams were asked to calculate the cost of their constructed towers (Cost $-\mathrm{C} 2)$. The time for completion of design and construction of each round was noted. As mentioned earlier, there was no specific time limit for finishing each round. However, it was anticipated that both rounds would be finished within 75 minutes, followed by a reflection and discussion period of approximately 20 minutes. 


\section{Simulation TEMPLATES DEVELOPED}

The authors of this paper had been participating in the online APLSO (Administering and Playing Lean Simulations Online) forum that provides an international platform for testing and development of lean simulations using the cloud-based software. Based on learnings gleaned during a 90-minute session playing with, and collecting plus-delta feedback fromparticipants during this forum, it was observed that, for enabling participants to experience the "aha moment" that makes simulations effective in illuminating lean principles, online lean simulations require convenient software platforms that participants can trust. Because of this, the team decided to use "Google Slides" for the templates when they played this simulation at the APLSO forum. Google Slides also offers the additional feature of privacy where templates can be accessed using Google Drive without players needing to volunteer their email addresses to software companies, avoiding concerns of later email spamming by software companies.

Three groups of slides were prepared for administration of this simulation.

\section{Instruction Slides}

The Instruction Slides provided information related to team composition, role playing, and instructions for playing Round 1 and Round 2. The general instructions given to participating teams were: (i) complete the tower without any voids between the shapes given within the space restrictions defined in the template and having $26 \mathrm{~cm}$ height and a base width of $12 \mathrm{~cm}$, and (ii) do not resize or change the shape of any pieces, with the one exception that shrinking (but no expanding) of the last placed piece was permitted. Dimensions of the pieces were provided in the TVD Game Playing Template as computational aids for the convenience of the players, in order to determine how best to address the final height and width specification of the two-dimensional tower.

\section{TVD Game Playing Templates}

There were two separate TVD Game Playing Templates to be used during Round 1 and Round 2. Both Round 1 and Round 2 consisted of Google Slides, and included an empty space for typing in the Owner's brief, uploading design proposals (Design Proposal - D1 and D2), and noting the RFIs exchanged between the Owner, Designer and Contractor. It also included a resource sheet containing pieces of various shapes and sizes, as well as a space for the construction of tower.

\section{Cost Sheet Template}

The Cost Sheet Template consisted of a table itemizing pre-defined cost rates of each shape, the number of shape variants used in final construction of the tower, and the total cost of tower. Both the TVD Game Playing Template and the Cost Sheet Template were made accessible by the facilitators by sending a link to Google Slides to all participants using the chat function of Google Meet. As a result of shared workspace, the hosting facilitators were able to observe the progress of each team on Google Slides as they played the simulation. This was especially helpful because the Screen Share function of Google Meet enabled facilitators to illustrate examples of team projects during the "big room" reflection and discussion stage of the exercise.

\section{Simulation TESTING}

The developed TVD online simulation was tested in a studio entitled "Construction Project Formulation and Appraisal." This studio was part of graduate course curriculum in Construction Engineering and Management at CEPT University. The primary objective 
behind this simulation was to provide an in-person experience to the students about collaborative working, early involvement of stakeholders in construction supply chain, and the process of designing to target cost. The instructor for this studio was assisted by two teaching assistants to administer this simulation.

The students involved in this studio were more familiar with the Google Meet platform for online meetings. Therefore, it was decided to use Google Meet to conduct the simulation. The students in the studio course were divided into teams of four to five. A total of 6 teams were formed and it was decided that each teaching assistant would be an observer for three teams while the instructor would oversee the overall simulation process by observing shared Google Slides templates, and addressing any specific questions raised. In total, 6 breakout rooms were created in Google Meet, one for each team. A few days before the actual playing of simulation, two power point presentations were shared with the students, including one by David Umstot and another by Prof. Lauri Koskela and Amit Kaushik. This helped the students in understanding the background of TVD. On the day of simulation, a conceptual foundation for Target Value Design was laid and the benefits delivered by TVD were highlighted with reference to the power point presentations. The discussion took approximately 20 minutes and was followed by a screen share of the "Instruction Template" in Google Slides. The students were asked if they had any questions about the simulation before they were given access to the "Game Playing Template" in Google Slides, and questions were clarified.

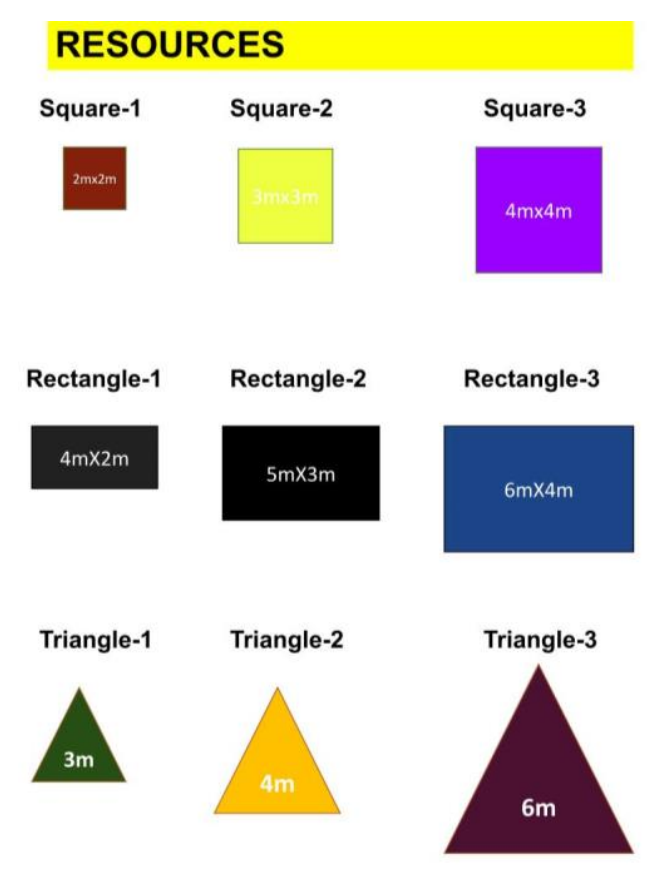

Resource Sheet

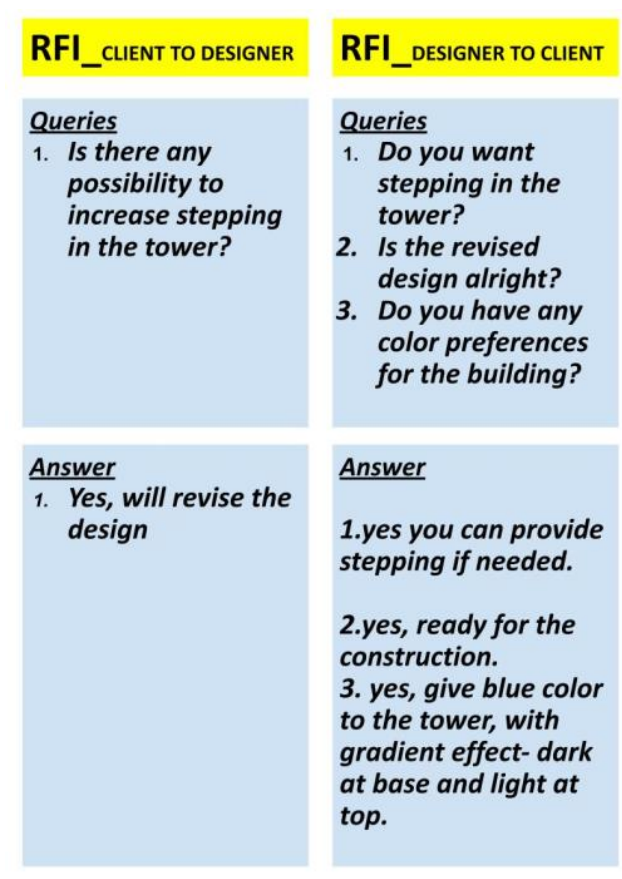

Filled RFI Sheet

Figure 1 - Templates of TVD Game

The "Game Playing Template" was then shared in Google Slides with the students and they were asked to join one of the breakout rooms created in Google Meet. During Round 1, the participant playing the role of the Owner could either type the strategic brief or upload an image containing a handwritten strategic brief into the workspace provided in the "TVD Game Playing Template." The participant playing the role of Designer prepared a hand-drawn sketch of the tower on a piece of paper conforming to the strategic brief and uploaded it in the workspace named "Design Proposal" in the "TVD Game Playing 
Template." During Round 1, the Designer often had to upload different interactions of the design until it was approved by the Owner. During Round 1, the teammates were not allowed to communicate verbally. A separate workspace in "TVD Game Playing Template" was included for the participants to raise their questions in the form of RFIs. The participants could type their questions in the space provided and the member of the team responsible could type back a response. Round 1 was completed in 45 minutes. At the end of Round 1, the students were asked to return to the "Main Google Meet Room." The "Cost Sheet" was then shared with students so they could benchmark the costs of the constructed tower, based on their designs from Round 1. The cost sheets had been preloaded with formulas to calculate the sum total costs of the constructed towers. The rationale of not sharing the "Cost Sheet" until after completion of Round 1 was to avoid any influence of cost during preparation of the "Owner Brief" and "Design Proposal D1". As the students filled their cost sheets, the teaching assistants captured information from the cost sheets of each group and populated a shared excel sheet for the class.

The excel sheet developed as part of TVD marshmallow tower simulation was used for the cost calculation for both the rounds (Rybkowski et al. 2016). This excel sheet consisted of three parts: 1) Establish Market Cost, 2) Establish Target Cost and 3) Design to Target Cost. A "Target Cost" was then declared by each team and noted on the spreadsheet. The instructor projected the excel sheet in the "Main Google Meet Room" and drew student attention toward the highest and lowest constructed tower costs. The instructor then calculated the market cost (average of construction cost of all towers). Allowable cost (the maximum cost to which the Owner could realistically build, determined by their financial resources and business case) was established by dropping the average cost of each tower by $20 \%$, with the help of the cost sheet. Market cost, allowable cost, target cost, and the actual costs achieved in each round is mentioned in Table 1. The teams were then asked to return to their respective breakout rooms for five minutes and brainstorm a declared target cost. The rationale for providing a very limited time window for brainstorming was to incentivize intense collaborative discussion and to avoid a scenario wherein teams start to actually design the tower with reference to cost. After all the teams re-assembled in the "Main Google Meet Room," each team openly declared its "target cost" and the average cost of all declared target costs was calculated in the excel sheet. Then, the teams were asked to return to their respective Google Breakout Room to play Round 2.

Table 1: Comparative Cost Statement

\begin{tabular}{|c|c|c|c|c|c|c|}
\hline & Team 1 & Team 2 & Team 3 & Team 4 & Team 5 & Team 6 \\
\hline Cost (C1) & 278 & 218 & 248 & 248 & 215 & 209 \\
\hline Market Cost & & &. .236 & & & \\
\hline Allowable Cost & $\ldots$. & 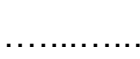 & $\ldots 189$ & & & $\ldots$ \\
\hline Declared Target Cost & 250 & 190 & 220 & 200 & 170 & 190 \\
\hline Average Target Cost & & & $\ldots 203$ & & & $\ldots$ \\
\hline Cost (C2) & 230 & 173 & 194 & 194 & 176 & 149 \\
\hline
\end{tabular}

All figures are in INR. 1 USD $=72$ INR.

During Round 2, the participants were instead permitted to finalize the design brief collaboratively and write or upload it on the TVD game-playing template. To foster collaboration and hasten finalization of the tower designs, the Round 2 permitted 
switching on of cameras and verbal communication between team members, and the Designer was permitted to show different iterations visually on camera to the Owner and the Contractor, seeking their feedback, before finalizing their team design (Design D2) - all the while keeping in mind the declared target cost and parameters specified in the design brief. During both Round 1 and Round 2, the workspace named "Construction Site" allowed the Contractor to construct the tower according to the finalized design by copying and pasting required shapes from a laydown area named "Resources" in the "TVD Game Playing Template." Participants completed Round 2 within approximately 30 minutes, after which all teams returned to the "Main Google Meet Room." Each team filled their respective cost sheet for Round 2 and the details were captured online by the teaching assistants in the shared excel sheet. The excel sheet was shared in the "Main Google Meet Room" and teams were asked to reflect on parameters such as total cost at the end of Round 2, total cost at the end of Round 1, and declared target costs. In addition to costs from various rounds, the excel sheet captured parameters such as time of completion of design and construction in Round 1 and Round 2, and the number of RFIs incurred during Round 1. The excel sheet helped participants develop an understanding of an overview of TVD concepts and goals, including market cost, allowable cost, and target cost. Figure 2 depicts the designs prepared by the students during Round 1 and 2, the constructed tower in each round, and the populated cost sheet.
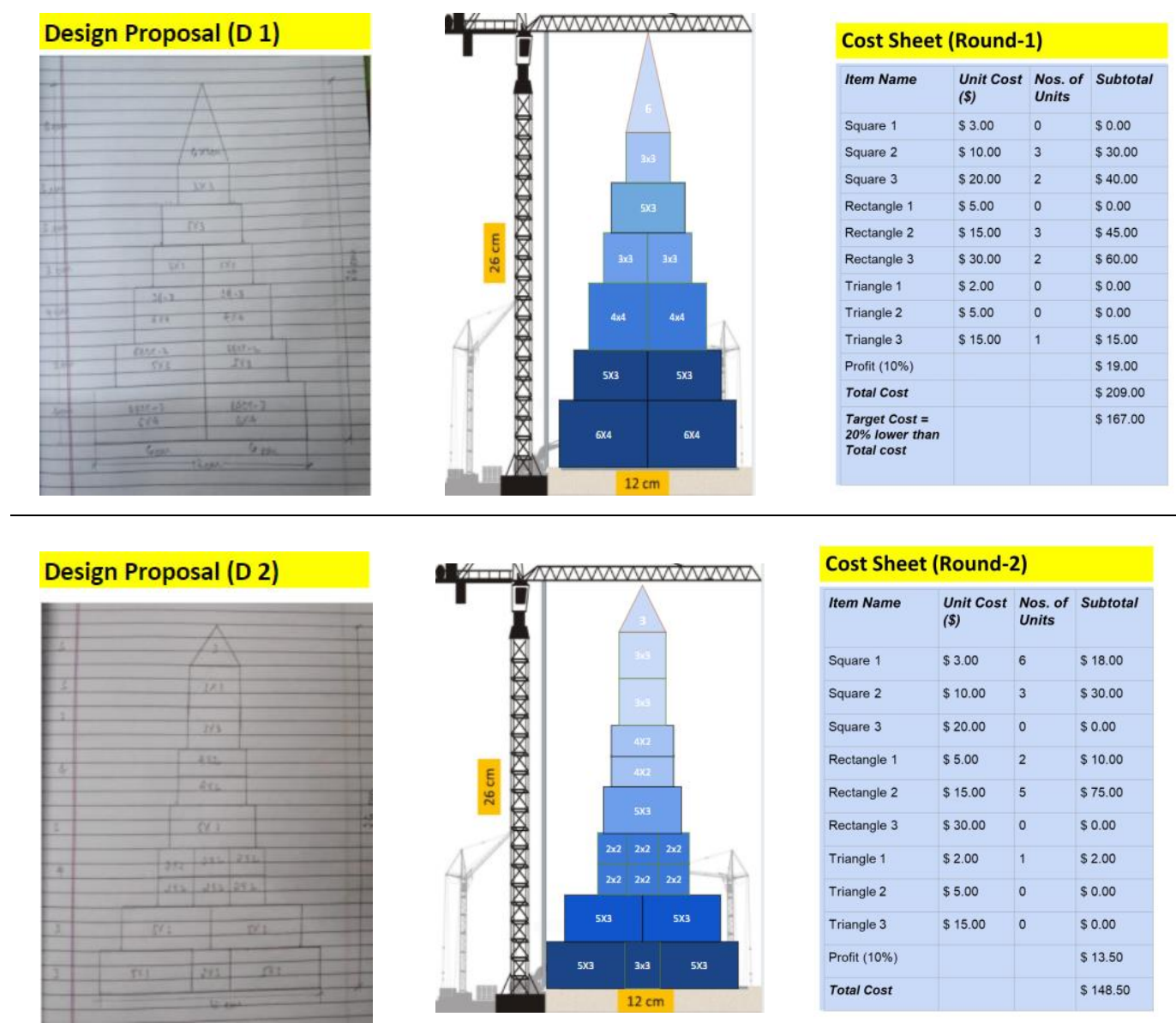

Figure 2- Designs prepared and actual towers constructed for Round 1 (above) and Round 2 (below) 


\section{POST SIMULATION DISCUSSION}

After Round 2, during the reflection phase of this game, students were asked to consider concepts such as their understanding of collaboration, rapid cost feedback, target costing, designing to target cost, working environment, relevance of learned concepts to practice and so on. Based on this discussion, it was evident that the simulation was effective in providing a first-hand experience of TVD concepts. The instructor also used the same evaluation questionnaire developed by Munankami (2012) to evaluate the effectiveness of this online TVD simulation. Originally, the evaluation questionnaire was developed to assess the effectiveness of the marshmallow tower TVD simulation, developed, and tested by Munankami (2012). Devkar et al. (2012) used the same questionnaire for evaluating the effectiveness of a TVD simulation exercise. It was also used by Musa et al. (2019) when the TVD simulation was administered to 24 practitioners in Nigeria to test their understanding of TVD. Therefore, for consistency, the authors chose to use the same evaluative questionnaire for their online simulation. The online platform provided by Google Forms was used for administering the questionnaire and was circulated among the students at the end of the reflection/ discussion session. The students were asked to immediately provide responses to the questionnaire, since learnings and their "aha moments" were likely still fresh in their minds at the end of the simulation. In the survey form, the students were asked to rate various parameters on a 5-point Likert scale: 5 (most effective) to 1 (least effective). The questionnaire consisted of 23 questions in total, and an analysis of the most relevant responses are as follows:
A. Mutual respect and trust
B. Mutual benefit and reward
C. Collaborative innovation and decision making
D. Early involvement of key partners
E. Intensified planning
F. Open communication
G. Owner is an active member of the team
H. Understanding the value of customer

Continuous estimating and budgeting through collaboration among team membersThere were total of 22 respondents to this questionnaire. Based on the analysis of responses to the questions (Figure 3), it was observed that the students had developed an enhanced understanding of "collaborative innovation and decision making." During the post simulation discussion, the students mentioned that Round 2 helped break down typically experienced boundaries between the Owner, Designer and Contractor. Involvement of key stakeholders assisted in a team's development of innovative towers not only met Owner's needs but also lowered the cost of construction. This collaborative decisionmaking helped the participants understand that a win-win situation was created in Round 2 , reflected in the observation that most respondents gave a higher rating (greater than or equal to 4) for the parameters of mutual trust and reward. Most notably, the analysis of questionnaire responses indicates that the concept of "target costing" was understood by the participants. Also, the efficacy of cost feedback in the design process to meet target cost and value expectations of the owner is reflected in the higher rating (equal to 5) received by the parameter "continuous estimating and budgeting through collaboration 
among team members." Additionally, the participants seemed aware of the efficacy of open communication, which was also rated higher (greater than or equal to 4). Given the richness of discussions between participants and the instructor during the reflection phase, as well as results from the questionnaire responses, it appears the online TVD simulation effectively served the purpose for which it was designed.

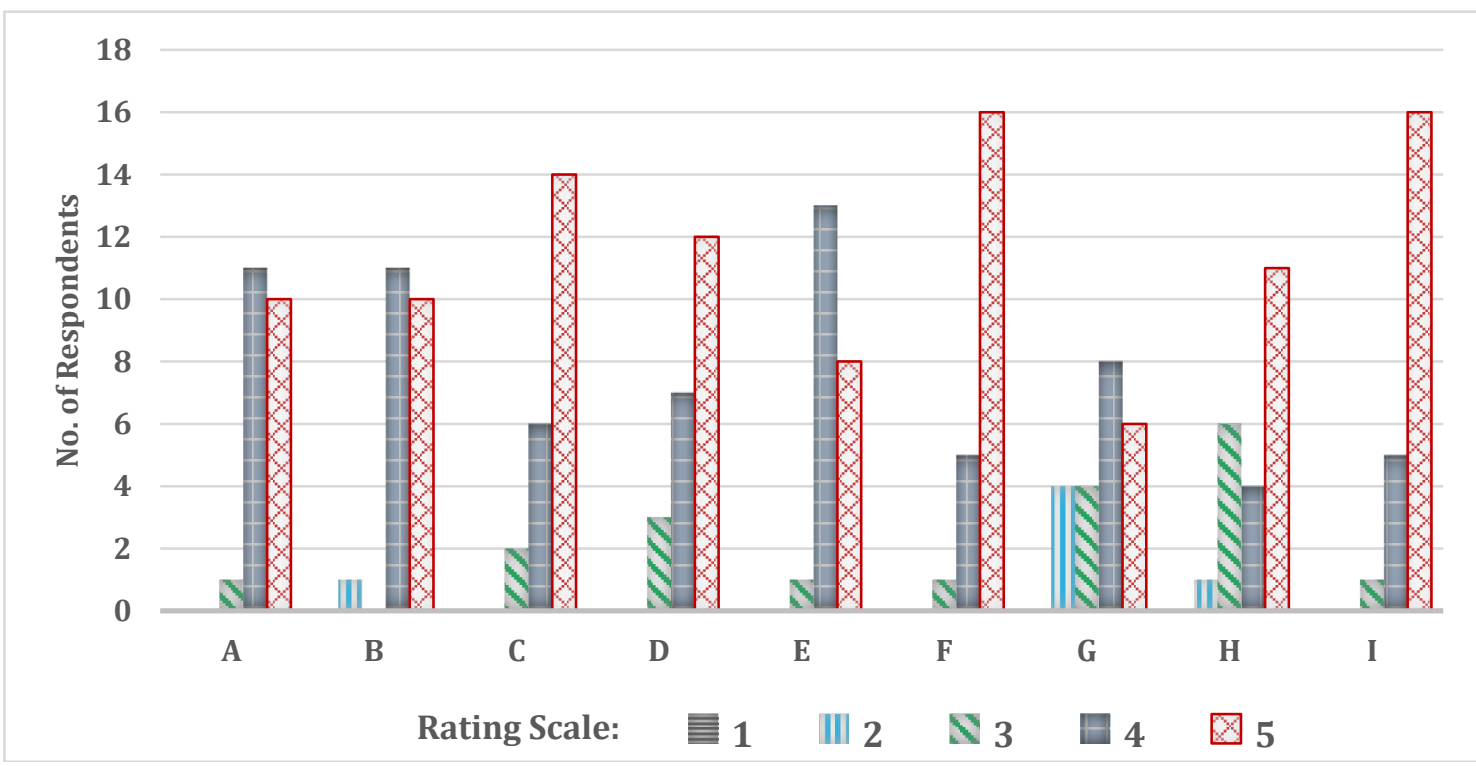

Figure 3 - Histogram showing participant's response to questions about the online TVD simulation

\section{CONCLUSION}

Target Value Design is a concept from lean construction which attempts to break boundaries created among three important construction supply chain actors, namely the Owner, Designer and Contractor. The adoption of this concept not only promises monetary gains but also cultural shifts in terms of collaboration, transparency and teamwork which is essential for efficient operations within the construction supply chain. In this context, the authors of this paper reported on the development and testing outcomes of an online TVD simulation. The development of the simulation involved creation of user-friendly instructions and templates with movable, shaped pieces to be used to build a two-dimensional tower. The simulation was administered on the Google Meet Platform and facilitated by the instructor and teaching assistants involved in development of this simulation. Responses from players were positive and appeared to indicate that key concepts critical to an understanding of TVD were being imparted. The online TVD simulation appears to have served as an effective replacement for the in-person simulation during the COVID-19 pandemic. Perhaps a more effective test of its efficacy will be whether or not this online version of the TVD simulation will continue to be played even after the pandemic has ended. The developed online TVD simulation has not explored the facet of "functionality" in terms of designs and cost effectiveness. However, it can be explored in the enhanced version of this simulation in future. Also, this simulation allows design and construction of tower in $2 \mathrm{D}$, however, more advanced software platforms can be used for design and visualization of tower construction in $3 \mathrm{D}$ with rapid cost feedback process. 


\section{REFERENCES}

Ballard, G. (2008). The lean project delivery system: An update. Lean Construction Journal, 2008, 1-19.

Ballard, G., and Rybkowski, Z. K. (2009). Overcoming the hurdle of first cost: action research in target costing. 1038-1047.

Devkar, G., Trivedi, J., and Pandit, D. (2019). Teaching target value design: A simulation. 27th Annual Conference of the International Group for Lean Construction, IGLC 2019, 479-490. doi.org/10.24928/2019/0244.

Do, D., Chen, C., Ballard, G., and Tommelein, I. D. (2014). Target value design as a method for controlling project cost overruns. 22nd Annual Conference of the International Group for Lean Construction: Understanding and Improving Project Based Production, IGLC 2014, 1(510), 171-181.

Jung, W., Ballard, G., Kim, Y.-W., and Han, S. H. (2012). Understanding of Target Value Design for Integrated Project Delivery with the Context of Game Theory. Construction Research Congress, 556-563.

Lee, H. W., Ballard, G., and Tommelein, I. D. (2012). Developing a Target Value Design protocol for commercial energy retrofits - Part 1. Construction Research Congress 2012: Construction Challenges in a Flat World, Proceedings of the 2012 Construction Research Congress, 2008, 1710-1719. doi.org/10.1061/9780784412329.172.

Miron, L. I. G., Kaushik, A., and Koskela, L. (2015). Target value design: The challenge of value generation. Proceedings of IGLC 23 - 23rd Annual Conference of the International Group for Lean Construction: Global Knowledge - Global Solutions, July, 815-825.

Munankami, M. B. (2012). "Development and testing of a simulation (game) to illustrate basic principles of Integrated Project Delivery and Target Value Design: A First Run Study," Graduate Thesis, Texas A\&M University, College Station, TX.

Musa, M., Pasquire, C., and Hurst, A. (2019). "Using TVD Simulation to Improve Collaboration." In: Proc. 27th Annual Conference of the International Group for Lean Construction (IGLC), Pasquire C. and Hamzeh F.R. (ed.), Dublin, Ireland, pp. 503514. doi.org/10.24928/2019/0268.

Rybkowski, Z. K., Forbes, L. H., and Tsao, C. C. Y. (2020). The evolution of Lean Construction education at US-based universities. Lean Construction, 387-407. doi.org/10.1201/9780429203732-19.

Rybkowski, Z. K., Munankami, M. B., Shepley, M. M., and Fernández-Solis, J. L. (2016). Development and testing of a lean simulation to illustrate key principles of target value design: A first run study. IGLC 2016 - 24th Annual Conference of the International Group for Lean Construction, 133-142.

Skillman, P. (2014) "Peter Skillman Marshmallow Design Challenge." https://www.youtube.com/watch?v=1p5sBzMtB3Q (March 25, 2021).

Umstot, D. (2016). Target Value Design (Delivery) An Overview [PowerPoint slides]. Umstot Project \& Facilities Solutions LLC. https://www.lcicongress.org/pdfs/MON7_Target_Value_Design.pdf. 